\title{
Framing faces: Frame alignment impacts holistic face perception
}

\author{
Kim M. Curby ${ }^{1,2} \cdot$ Robert Entenman ${ }^{3}$
}

Published online: 2 September 2016

(C) The Psychonomic Society, Inc. 2016

\begin{abstract}
Traditional accounts of face perception emphasise the importance of the prototypical configuration of features within faces. However, here we probe influences of more general perceptual grouping mechanisms on holistic face perception. Participants made part-matching judgments about composite faces presented in intact external oval frames or frames made from misaligned oval parts. This manipulation served to disrupt basic perceptual grouping cues that facilitate the grouping of the two face halves together. This manipulation also produced an external face contour like that in the standard misaligned condition used within the classic composite face task. Notably, by introducing a discontinuity in the external contour, grouping of the face halves into a cohesive unit was discouraged, but face configuration was preserved. Conditions where both the face parts and the frames were misaligned together, as in the typical composite task paradigm, or where just the internal face parts where misaligned, were also included. Disrupting only the face frame similarly disrupted holistic face perception as disrupting both the frame and face configuration. However, misaligned face parts presented in aligned frames also incurred a cost to holistic perception. These findings provide support for the contribution of general-purpose perceptual grouping mechanisms to holistic face perception and are presented and discussed in the context
\end{abstract}

Kim M. Curby

kim.curby@mq.edu.au

1 Department of Psychology, Macquarie University, Building C3A, Sydney, NSW 2109, Australia

2 Centre for Elite Performance, Expertise, and Training, Macquarie University, Sydney, NSW, Australia

3 Department of Psychology, Temple University, Philadelphia, PA, USA of an enhanced object-based selection account of holistic perception.

Keywords Face perception · Object-based attention . Grouping and Segmentation

Accounts of face perception have traditionally highlighted and emphasized the importance of the prototypical configuration of features within faces. Facial configuration has been suggested to be critical in triggering or allowing access to facespecific mechanisms (e.g., McKone, Kanwisher, \& Duchaine, 2007; Tsao \& Livingstone, 2008) and in structuring and providing the basis of a norm and/or template underlying holistic face processing (Diamond \& Carey, 1986; McKone et al., 2007; Rhodes \& Jeffery, 2006; Rossion, 2013). Support for the importance of facial configuration comes from studies demonstrating that disrupting the configuration of facial features, via a variety of different manipulations, impairs holistic processing (e.g., Tanaka \& Farah, 1993; Tanaka \& Sengco, 1997; Yin, 1969; Young, Hellawell, \& Hay, 1987). However, these manipulations of facial configuration also typically result in a disruption to the coherence of the face as a perceptual unit and thus the degree to which the facial parts are grouped together at a more basic perceptual level. Thus, it is unclear to what degree this disruption to perceptual grouping, rather than the change in the configuration of the facial features, drives the reduction in holistic face perception. Here we examine whether we can attenuate a classic marker of holistic face perception by disrupting perceptual grouping, while keeping the configuration of features within the face intact.

The holistic nature of face processing is one of the most striking differences between the processing of face and nonface objects. Holistic processing refers to how facial features are obligatorily processed in the context of their relations with 
other features, creating more efficient representations (Farah, Wilson, Drain, \& Tanaka, 1998; Tanaka \& Farah, 1993; Tanaka \& Sengco, 1997) but also rendering it difficult for the person to ignore irrelevant face parts while attending to relevant parts (Gauthier, Curran, Curby, \& Collins, 2003). This processing style contrasts with the more "feature-based" processing typical for non-face objects, where object features are processed more independently.

A number of classic markers or indices of holistic processing have been established within the face recognition literature (e.g., Tanaka \& Sengco, 1997; Yin, 1969; Young et al., 1987). A key feature of these markers is that they appear to be dependent on the configuration of the features within the face being intact. One of the most robust is revealed in the composite task, which demonstrates people's obligation to process intact faces as wholes: when making a judgment about one half of the face, they experience interference from the other, task-irrelevant half. This has led to holistic perception, especially as revealed in the composite face task, being described as a failure of selective attention to parts (Richler, Tanaka, Brown, \& Gauthier, 2008). The degree to which manipulations of the task-irrelevant part impacts judgments about the task-relevant part provides an index of holistic perception. For example, holistic processing can be indexed via the composite effect: the cost to identifying part of a face (e.g., the top half) when it is aligned with the bottom half of a different face, relative to when these parts are misaligned (which disrupts holistic perception). The disruption to holistic face perception by misalignment is considered a consequence of the disruption to the configuration of features within the face.

Part-matching judgments about composite faces are also influenced by the compatibility of the task-irrelevant face parts (i.e., whether it would require the same [congruent] or a different [incongruent] response as that for the task-relevant part; congruency effect) (Gauthier et al., 2003). The congruency effect, which serves as another index of participants' inability to selectively process only the task-relevant face parts, is also attenuated for misaligned face composites, providing further support for the importance of an intact facial configuration for holistic face perception. In fact, the degree to which the congruency effect is modulated by alignment is a key feature of holistic processing (Richler, Bukach, \& Gauthier, 2009).

However, examples where holistic face perception is modulated in the absence of a disruption to the configuration of the face raise questions about the degree to which holistic processing is tied to face configuration. For example, indices of holistic processing can be modulated by manipulating observers' emotional states (Curby, Johnson, \& Tyson, 2012). Inducing a negative emotional state resulted in a drop in holistic face perception. The degree to which indices of holistic face perception were reduced by the induction of a negative emotional state could be predicted by the degree of change observers reported in their mood at an individual level. Further, the degree to which holistic face perception was facilitated by a positive mood induction could also be predicted by the effectiveness, at an individual level, of the positive mood induction in inducing a positive mood. Notably, positive emotional states have been suggested to broaden the distribution of attention, while negative emotional states are suggested to do the opposite (Fredrickson \& Branigan, 2005). Thus, holistic perception may depend on the adoption of an appropriate, broader attentional strategy. This finding is consistent with a potential role of attention in supporting holistic perception of faces.

Clues to the mechanisms that drive holistic processing might be found in the literature on 'object-based' attention. Intriguingly, object-based attention appears to result in phenomena not unlike the failure of selective attention revealed within the composite task. Attention is allocated to objects in an "object-based" manner with the object being the unit of selection. A typical marker of object-based attention is that when attention is directed to part of an object, other parts of that object enjoy a processing advantage (Egly, Driver, \& Rafal, 1994). This is referred to as a same-object advantage. However, this "advantage" can also be a cost under altered task conditions. For example, flanker interference is greater when non-targets flanking a target are perceived to be on the same, compared to a different, object (Kramer \& Jacobson, 1991). In the case of the composite face task, object-based attention would result in attention "spilling" over to the task-irrelevant face part, producing performance costs when the task-irrelevant part contains misleading information. In this way, the automatic spreading of attention to (Hollingworth, Maxcey-Richard, \& Vecera, 2012; Richard, Lee, \& Vecera, 2008), or prioritization of (Shomstein \& Yantis, 2002), the task-irrelevant regions of the same object can effectively produce a failure of selective attention not unlike that observed in the context of the composite face task. Thus, it is possible that the holistic processing effects documented in the composite face task are supported by, at least in part, object-based attention.

The perception of "objecthood" is essential for object-based attention. Objecthood refers to the perception of a shared structure and/or identity across visual features or parts (Palmer, 1977), and it is strongly influenced by perceptual grouping cues such as connectedness, similarity, and good continuation of form (Palmer, 1977). Importantly, indexes of object-based attention are strongly influenced by manipulations of perceptual grouping cues designed to impact the perception of objecthood (Matsukura \& Vecera, 2006). It is noteworthy that many of the manipulations that have been shown to disrupt holistic face perception are also known to impact the perception of objecthood, such as manipulations of depth-plane (Nakayama, Shimojo, \& Silverman, 1989; Taubert \& Alais, 2009) or inversion of highly familiar stimuli (Vecera \& Farah, 1997; Yin, 1969). For example, Vecera and Farah (1997) demonstrated that 
inversion of letter stimuli, which, like faces, are a highly familiar category for which observers typically have considerable expertise processing, disrupted image segmentation. Notably, image segmentation is a critical precursor to the perception of objecthood. In addition, the misalignment of face parts, one of the most widely used manipulations to disrupt holistic face perception, disrupts the Gestalt grouping cue of good continuation of contours (among others), most obviously in terms of the external contour of the face. Thus, it is possible that the perception of objecthood, via its role in supporting object-based attention, is critical for the holistic perception of faces.

The potential role of basic factors determining perceptual grouping and the perception of objecthood in supporting holistic perception has also been highlighted by evidence that strong Gestalt grouping cues can drive face-like holistic perception of non-face objects (Zhao, Bulthoff, \& Bulthoff, 2016). Specifically, face-like holistic perception was found for nonface line patterns that were strong in Gestalt grouping cues. However, once the Gestalt information in these stimuli was weakened, by converting them to dot (line) patterns, the degree to which they were processed holistically was markedly reduced. These findings are also generally consistent with the proposed role of Gestalt cues in supporting holistic perception.

Consistent with the suggested importance of intact perceptual grouping for holistic face perception, we have also provided evidence that manipulations that weaken the objecthood of stimuli, and thus object-based selection of the stimulus, impair indices of holistic perception of face, but not non-face, stimuli (Curby, Entenman, \& Fleming, 2016; Curby, Goldstein, \& Blacker, 2013). Specifically, when composite faces are presented in the context of perceptual cues that disrupt the perceptual grouping of the faces parts, namely when the parts are presented on differently colored and misaligned rectangles, holistic perception is impaired. However, one limitation of this study was that the effects were based on the introduction of cues discouraging the grouping of the framing elements, rather than the face parts themselves. This was necessary as the importance of face configuration in driving holistic face perception can not be teased apart from the potential contribution of more general mechanisms related to objecthood and object-based attention, unless the latter were manipulated while the internal configuration of facial features remained intact. However, as a consequence, the manipulations employed in our previous studies were indirect. Here we aim to more directly assess the contribution of a disruption to objecthood in producing the drop in holistic processing with misalignment in the composite face task.

\section{Experiment 1}

Experiment 1 examines the proposal that the drop in holistic processing when face parts are misaligned, as measured by the congruency effect, is driven, in part, by a disruption to the perceptual grouping of the face parts, rather than just to the configuration of features within the face. As noted earlier, misaligning the face parts disrupts the good continuation of the external face contour, an important Gestalt grouping cue that supports the perception of objecthood of the face. The robust drop in indices of holistic perception with misalignment may be a result of disrupted perceptual grouping, or more specifically its consequences for object-based attention. These predictions were examined using a modified composite face task where the framing contours of the face stimuli were misaligned, but the configuration of the internal features, that is, the spatial arrangement of the eyes, nose, and mouth, remained intact. Notably, here the framing contour refers to the contour of the shape within which the face stimulus is embedded, not the natural contour of the face (e.g., the jaw line). To provide baselines for comparison, the standard conditions where the framing contour and the internal features were either both aligned or both misaligned were also included. If object-based attention plays a key role in producing holistic perception of faces, it is predicted that disrupting only the external framing contour of the face (and thus object-based selection of the face) will attenuate holistic perception similarly to when both the external contour and internal features are misaligned. Alternatively, if object-based selection does not contribute to holistic perception of faces, holistic perception should remain intact, even when object-based selection is disrupted, providing the familiar face configuration is preserved, as in the case when only the framing contour is disrupted.

\section{Methods}

Participants Thirty-nine (23 female, Mean age $=21.5$ years, $\mathrm{SD}=2.3$ ) undergraduate psychology students participated in this study for course credit. All individuals reported having normal or corrected to normal vision.

Stimuli The stimuli consisted of 20 greyscale front-view images of male (ten) and female (ten) faces wearing neutral expressions from the Karolinska Directed Emotional face (KDEF) database (Lundqvist, Flykt, \& Ohman, 1998). Using Photoshop, the faces were placed in an oval frame that covered their hair and other external features. The faces and frames $\left(7.8^{\circ} \times 7.8^{\circ}\right)$ were then divided into two halves along a horizontal plane aligned with the bridge of the nose. Two additional versions of the stimuli were made: (1) where the two frame halves, but not the faces halves were misaligned by $0.68^{\circ}$ by shifting the bottom and tops halves of the frames by $0.34^{\circ}$ in opposite directions, and (2) where both the faces parts and the frames were misaligned together. Note that the images were adjusted so that only the internal framing contour was misaligned, but not the external contour of the frame (see Fig. 1 for example images). 


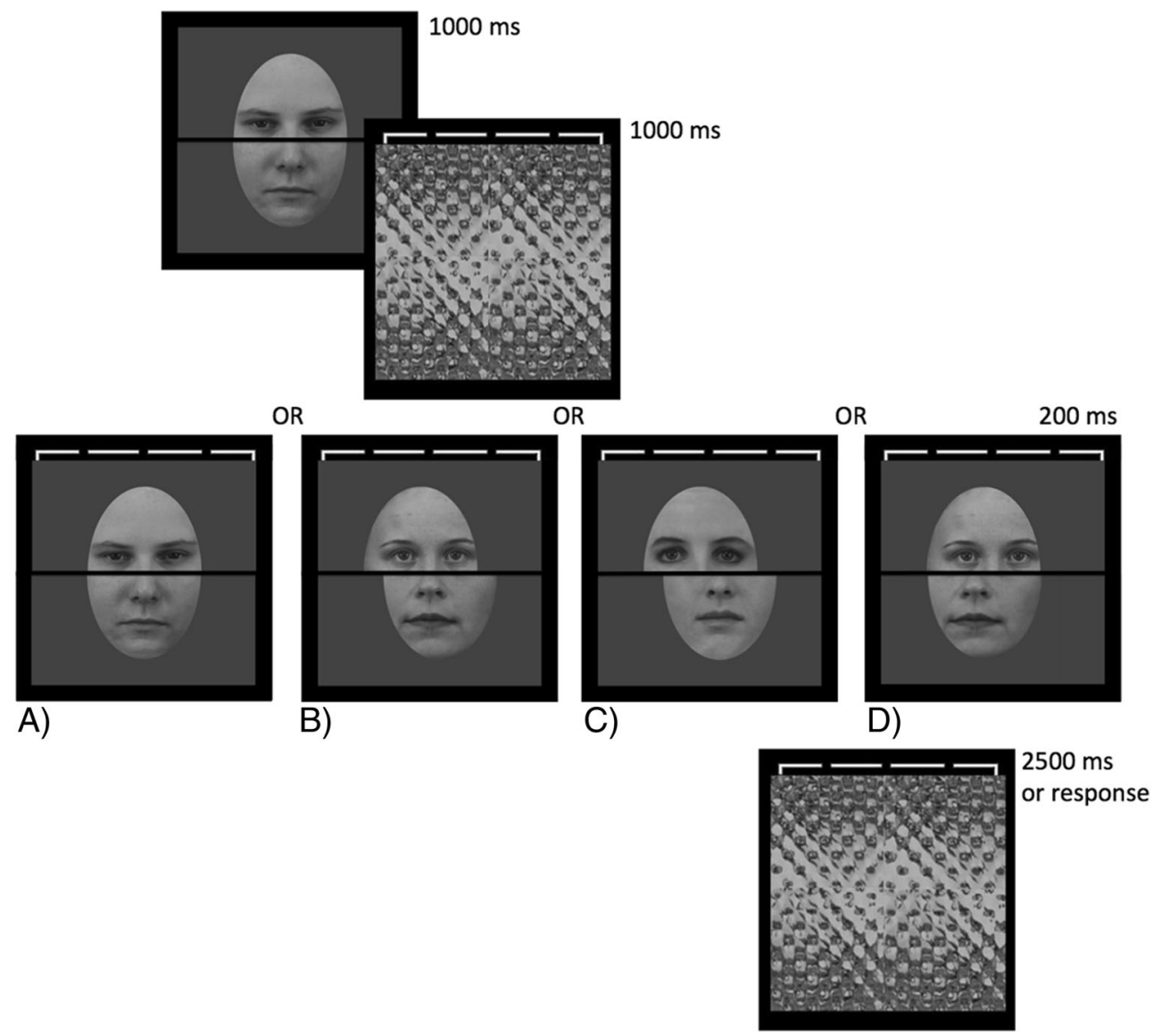

Fig. 1 Trial structure used for the modified composite face task. The aligned $[(\mathbf{A})$ and $(\mathbf{B})]$ and misaligned $[(\mathbf{C})$ and $(\mathbf{D})]$ face parts either appeared in aligned $[(\mathbf{A})$ and $(\mathbf{D})]$ or misaligned $[(\mathbf{B})$ and $(\mathbf{C})]$ frames. (Note, the condition where the misaligned face was presented in an aligned frame [i.e., (D)] only appeared in Experiment 2.) A condition was also included where the task-relevant part was presented in

Procedure Participants performed a modified version of the composite face task. As in the standard composite task, face composites were created by combining the top of one face with the bottom of another face. The top and bottom parts were presented so that they either appeared aligned or misaligned. However, if the faces parts were aligned, they either appeared in an oval frame in which the top and bottom frame parts were also aligned (Fig. 1A) or in a frame that was misaligned (Fig. 1B). In the cases where the face parts were misaligned, the frames were also misaligned (Fig. 1C). The same face identities were used in all the conditions, thus the stimuli across the conditions only differed in terms of the framing and/or configuration (alignment) of the top and bottom parts. Each trial started with a central fixation dot $(1,000 \mathrm{~ms})$, followed by a composite (chimeric) face comprising of the top and bottom parts of different faces $(1,000 \mathrm{~ms})$. This was masked by a textured pattern (for 1,000 ms) that also contained either a bracket around the top or bottom part indicating which part was the task-relevant part. Following the mask, a second face and the bracket cue were briefly presented isolation (not shown). A dashed bracket served as the cue in each trial to indicate which face part (top or bottom) the participant should make a same/different judgment on. In the example, the bracket is around the top half, indicating the matching judgment should be performed on the top part

(200 ms), followed again by the textured pattern and bracket cue (for 2,500 ms or until response). Participants' indicated via a key press whether the cued half (top or bottom) of the second face was the same or different in identity to that of the first face. Participants completed 16 blocks of 16 trials containing faces in either aligned (four blocks) or misaligned face frames (eight blocks). For blocks were the frame parts were misaligned, the block either contained trials where the internal face parts were misaligned, consistent with the frame, or aligned (four blocks of each were included). The order of blocks was randomized for each participant and participants were offered a break every 32 trials. Within each block, for half of the trials the same/different relationship between the task-irrelevant (non-cued) face parts in the two faces was congruent with the relationship between the task-relevant (cued) parts. In other words, in congruent trials, if the taskrelevant parts differed between the two faces, thus rendering the correct response for the trial "different," the task-irrelevant face parts also differed. In the other half of the trials within a block, the same/different relationship between the task- 
irrelevant (non-cued) face parts in the two faces was incongruent with the relationship between the task-relevant (cued) parts. For example, if the task-relevant parts differed between the two faces, thus again rendering the correct response for the trial "different," the task-irrelevant face parts were the same. An isolated part condition (four blocks) was also included where only the task-relevant part of the second face was presented. Participants completed a total of 256 face trials, consisting of 64 trials where a face part was presented in isolation, and 96 of each congruent and incongruent trials spread evenly across the three main experimental conditions (faces aligned and frames aligned, faces aligned and frames misaligned, and faces misaligned and frames misaligned).

Analysis Given that the purpose of the study was to examine the contribution of the disruption to the external contour to the robustly documented drop in holistic processing observed when face parts are misaligned, only participants who had a holistic processing index $\left(\mathrm{d}^{\prime}\right.$ congruent $-\mathrm{d}^{\prime}$ incongruent $) \geq 0$ in the baseline, aligned face and aligned frame, condition were included. This resulted in data from one participant being excluded.

\section{Results}

Sensitivity analysis A 3 (alignment; aligned face/frame, misaligned face/frame, aligned face/misaligned frame $) \times 2$ (congruency; congruent, incongruent) ANOVA performed on the sensitivity (d') scores revealed a main effect of congruency, $\mathrm{F}(1,37)=90.16, \mathrm{p} \leq .0001, \eta_{\mathrm{p}}^{2}=.71$. The main effect of alignment failed to reach significance, $\mathrm{F}(2,74)=2.65, \mathrm{p}$ $=.078, \eta_{\mathrm{p}}^{2}=.07$. However, there was a significant interaction between congruency and alignment, $\mathrm{F}(2,74)=6.43, \mathrm{p}=.003$, $\eta_{\mathrm{p}}^{2}=.15$. Scheffe post-hoc tests revealed a significant effect of congruency in all three alignment conditions, all ps<.0001. However, the size of this effect, an index of holistic perception, varied considerably across conditions (see Fig. 2A).

Planned paired t-tests (2-tailed) performed on indices of holistic processing $\left(\mathrm{d}^{\text {' }}\right.$ congruent $\left.-\mathrm{d}^{\text {'incongruent }}\right)$ to address our specific hypotheses revealed the expected drop in holistic processing between the aligned face/frame and misaligned face/frame conditions, $\mathrm{t}(37)=3.73, \mathrm{p}<.001$, Hedges's $g_{a v}=.68^{1}$. There was also a significant drop in holistic processing when just the frame was misaligned (i.e., aligned face/misaligned frame condition) relative to when both the face parts and frames are aligned, $t(37)=$ $2.24, p=.032$, Hedges's $g_{a v}=.44$. However, there was no significant difference in holistic processing between when just the frame was misaligned and when both the face and

\footnotetext{
${ }^{1}$ Effect sizes were calculated following the procedure outlined in Lakens (2013).
}
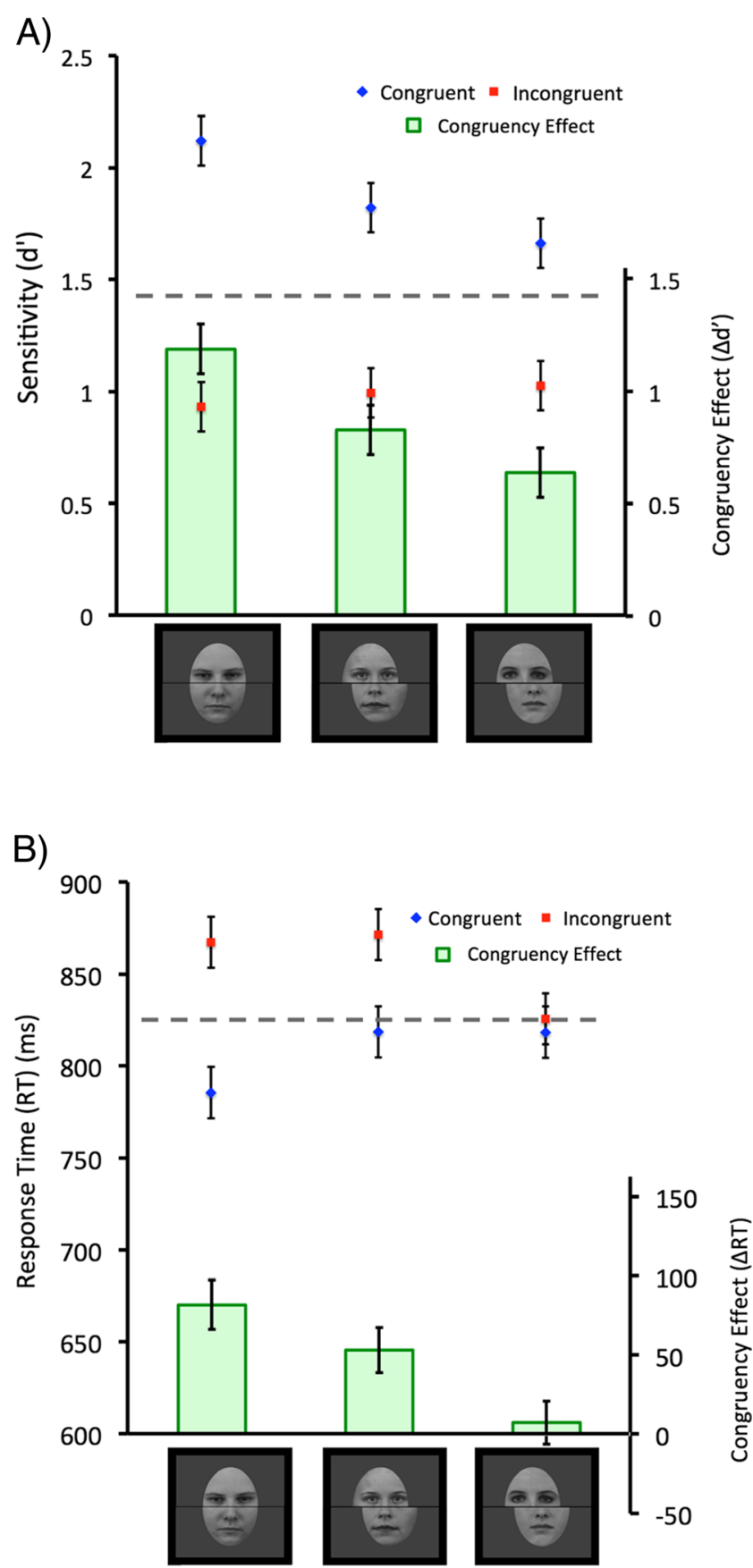

Fig. 2 (A) Mean sensitivity ( $\mathrm{d}^{\prime}$ ) and (B) response time for the congruent (diamonds) and incongruent (squares) conditions, and the resulting index of holistic perception (congruency effect, filled bars, reflecting the difference between these conditions) in Experiment 1. The dashed line shows the baseline performance level when only the task-relevant face part was presented. Holistic perception of aligned faces appearing in a misaligned face frame was reduced relative to that for faces presented in a aligned (intact) face frame. In the sensitivity (d') data, holistic perception in this condition was similar to that observed for faces where both the face parts and the frame were misaligned. Error bars represent standard error values

frames were misaligned, $\mathrm{t}(37)=1.20, \mathrm{p}=.237$, Hedges's $\mathrm{g}_{\mathrm{av}}=.25$, as in the standard composite face misaligned condition. 
Response time analysis A 3 (alignment; aligned face/frame, misaligned face/frame, aligned face/misaligned frame) $\times 2$ (congruency; congruent, incongruent) ANOVA was performed on the response time data from correct trials to aid in the interpretation of the analysis of the sensitivity (d') data. This analysis revealed a main effect of congruency, $F(1,37)=$ $28.04, \mathrm{p} \leq .0001, \eta_{\mathrm{p}}^{2}=.43$. There was no main effect of alignment, $\mathrm{F}(2,74)=1.52, \mathrm{p}=.226, \eta_{\mathrm{p}}^{2}=.04$. However, there was a significant interaction between congruency and alignment, $F(2,74)=7.19, p=.001, \eta_{p}^{2}=.16$. Scheffe post-hoc tests revealed a significant effect of congruency in face/frame aligned condition and in the face aligned/frame misaligned condition (both ps<.0015), but not in the face/frame misaligned condition ( $p>05$ ). Again, the size of this effect, an index of holistic perception, varied considerably across conditions (see Fig. 2B).

Planned paired t-tests (2-tailed) performed on RT indices of holistic processing (i.e., $\mathrm{RT}_{\text {congruent }}-\mathrm{RT}_{\text {incongruent }}$ ) revealed a drop in holistic processing between the aligned face/frame and misaligned face/frame conditions, $\mathrm{t}(37)=3.57, \mathrm{p}=.001$, Hedges $\mathrm{s}$ gav $=.82$. The change in holistic processing when just the frame was misaligned (i.e., aligned face/ misaligned frame condition), relative to when both the face parts and frames were aligned, did not reach significance, $t(37)=1.51$, $\mathrm{p}=.14$, Hedges $\mathrm{s}$ gav $=0.31$. However, there was a significant difference in holistic processing between when just the frame was misaligned (i.e., aligned face/ misaligned frame condition) compared to when both the face and frames were misaligned, $t(37)=2.35, p=.024$, Hedges $\mathrm{s}$ gav $=.52$, as in the misaligned condition in the standard composite task. Importantly, the general pattern of findings were not unlike that found in the analysis of the sensitivity data, that is, with the mean holistic processing index for the aligned face/ misaligned frame condition being at an intermediate level between the aligned face/frame and misaligned face/frame conditions, inconsistent with the presence of a speed accuracy trade-off.

Performance in the isolated part condition, that is, where only the task-relevant part was presented, generally fell numerically between performance in the conditions where a congruent or an incongruent task-irrelevant part appeared. This was the case for both sensitivity (d) and response time (RT) measures (see Fig. 2). This is consistent with the congruency effect being a product of both interference from incongruent parts and facilitation from congruent parts, although the contribution of each varied across conditions.

\section{Discussion}

The attenuation of holistic face perception by the disruption of basic perceptual cues grouping the face parts together, namely the good continuation of the external face contour, is consistent with the suggestion that perceptual grouping mechanisms contribute to holistic face perception effects. It is noteworthy that the external contour in the misaligned frame condition is the same as that in the standard misaligned condition in the composite task used to index holistic perception. However, unlike in the typical misaligned condition, the configuration of the features within the face remained intact. Strikingly, the sensitivity data revealed that the attenuation of holistic processing after disrupting the perceptual grouping of the face parts, but not their configuration, was comparable to that when both perceptual grouping and facial configuration was disrupted. Although, the response time data revealed greater holistic processing when only perceptual grouping, and not configuration, was disrupted.

\section{Experiment 2}

While we have demonstrated that disrupting perceptual grouping of the face parts can disrupt holistic perception, the degree to which perceptual grouping supports holistic perception is still unclear. Specifically, the absence of a condition in Experiment 1 where the external contour was intact, but the internal configuration of the facial features was disrupted, renders it difficult to tease apart the role of perceptual grouping cues and face configuration in supporting holistic face perception. Experiment 2 addresses this limitation by including a condition where the misaligned face parts are placed within an intact (aligned) face frame. The inclusion of this condition allows us to more fully assess the importance of the good continuation of the external face contour in supporting holistic perception effects. In addition, by including the same conditions as in Experiment 1, Experiment 2 not only has a full design where all combinations of frame and face alignment conditions are included, but it also allows us to assess the replicability of the effects reported in Experiment 1.

\section{Methods}

Participants Thirty (21 female, Mean age $=22.3$ years, $\mathrm{SD}=$ 7.1) undergraduate psychology students at Macquarie University participated in this study for course credit. All individuals had normal or corrected to normal vision. An a priori power analysis performed, based on the effect size of the congruency $\times$ alignment interaction in Experiment 1, indicated that a minimum of 22 participants would be required to achieve $95 \%$ power. Additional participants were allowed to sign-up for the study to allow for the failure of scheduled participants to show and/or data loss due to poor performance.

Stimuli The stimuli were the same as that used in Experiment 1 , except an additional version of the stimuli were created where the top and bottom halves of the composite faces were misaligned but were then placed in an aligned frame (see 
Fig. $1 \mathrm{D})$. The frame and misalignment procedure used to create these stimuli was the same as that used in Experiment 1.

Procedure and analysis The experimental procedure was the same as that in Experiment 1 except an additional condition was added where the misaligned face was presented in an aligned frame. Applying the same exclusion criteria as in Experiment 1 resulted in four participants being excluded.

\section{Results}

Sensitivity analysis A 2 (face alignment; aligned, misaligned) $\times 2$ (frame alignment; aligned, misaligned) $\times 2$ (congruency; congruent, incongruent) ANOVA was performed on the sensitivity (d') scores. This analysis revealed a main effect of congruency, $F(1,25)=90.55, p \leq .0001, \eta_{p}^{2}=.78$, but no main effect of face, $F(1,25)=.35, \mathrm{p}=.561, \eta_{\mathrm{p}}^{2}=.014$, or frame, $\mathrm{F}(1,25)=.05, \mathrm{p}=.834, \eta_{\mathrm{p}}^{2}=.0018$, alignment. However, there was a significant interaction between congruency and face alignment, $F(1,25)=9.66, p<.005, \eta_{p}^{2}=.28$. There was also an interaction between congruency and frame alignment, $\mathrm{F}(1,25)=4.26, \mathrm{p}<.050, \eta_{\mathrm{p}}^{2}=.15$. Scheffe posthoc tests revealed a significant effect of congruency in all face/ frame alignment conditions, all ps $<.002$. However, as in Experiment 1, the size of this effect, an index of holistic perception, varied considerably across conditions (see Fig. 3A).

To explore the basis of the interactions between congruency and face or frame alignment, planned paired t-tests (2-tailed) were performed on indices of holistic processing $\left(\mathrm{d}^{\text {' }}\right.$ congruent ${ }^{-}$ $\mathrm{d}^{\prime}$ incongruent). These tests revealed the expected drop in holistic processing when both the face parts and the frames were misaligned compared to when they were both aligned, $t(25)$ $=4.01, \mathrm{p}=.0004$, Hedges's $\mathrm{g}_{\mathrm{av}}=.90$. The expected drop in holistic processing when just the frame was misaligned (i.e., aligned face/misaligned frame condition) relative to when both the face parts and frames are aligned, was marginally significant $\mathrm{t}(25)=1.95, \mathrm{p}=.062$, Hedges's $\mathrm{g}_{\mathrm{av}}=.48$, consistent with the main findings from Experiment 1 . There was also a drop in holistic processing when just the face parts were misaligned (i.e., misaligned face/aligned frame condition) relative to when both the face parts and frames are aligned, $\mathrm{t}(25)=2.99, \mathrm{p}=$ .006 , Hedges's $g_{\mathrm{av}}=.77$. Notably, indices of holistic perception in both these conditions, that is, where only the frame, $\mathrm{t}(25)=1.40, \mathrm{p}=.172$, Hedges's $\mathrm{g}_{\mathrm{av}}=.42$, or only the faces parts where misaligned, $\mathrm{t}(25)=.36, \mathrm{p}=.719$, Hedges's $\mathrm{g}_{\mathrm{av}}=$ .09 , did not differ from that when both the face and frame were misaligned, as in the standard composite face misaligned condition, nor did they differ between themselves, $\mathrm{t}(25)=1.25, \mathrm{p}=$ .222 , Hedges's $\mathrm{g}_{\mathrm{av}}=.31$.

Response time analysis A 2 (face alignment; aligned, misaligned) $\times 2$ (frame alignment; aligned, misaligned $) \times 2$ (congruency; congruent, incongruent) ANOVA was
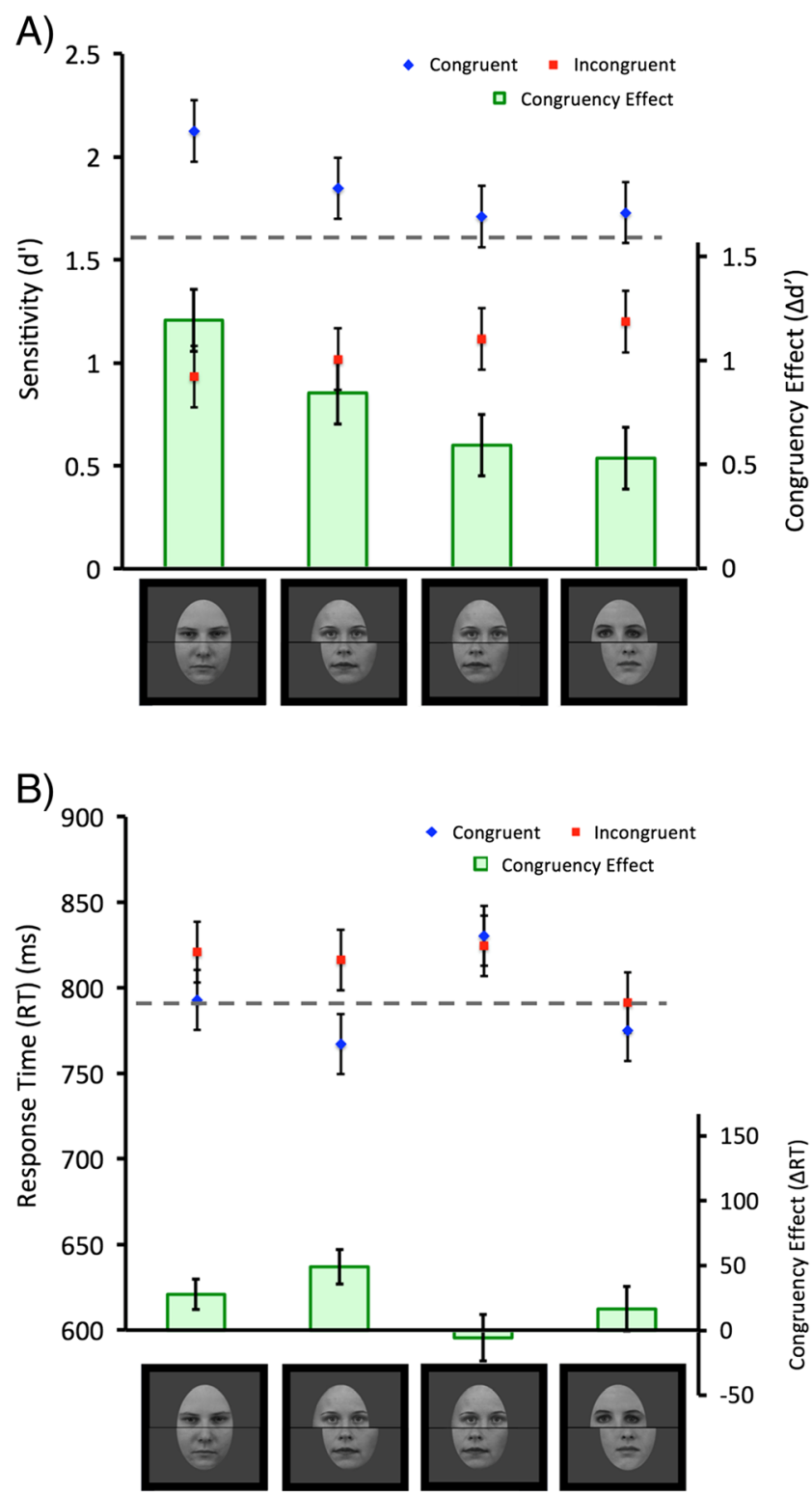

Fig. 3 (A) Mean sensitivity (d') and (B) response time for the congruent (diamonds) and incongruent (squares) conditions, and the resulting index of holistic perception (congruency effect, filled bars, reflecting the difference between these conditions) in Experiment 2. The dashed line shows the baseline performance level when only the task-relevant face part was presented. As found in Experiment 1, in the sensitivity (d') data, holistic perception of aligned faces appearing in a misaligned face frame was reduced relative to that for faces presented in a aligned (intact) face frame. However, the aligned frame was not able to facilitate holistic perception of misaligned face parts; holistic perception of misaligned faces presented in aligned or misaligned frames was similarly poor. Error bars represent standard error values

performed on the response time data from correct trials to aid in the interpretation of the analysis of the sensitivity (d') data. This analysis revealed a main effect of congruency, $F(1,25)=10.84, p=.003, \eta_{p}^{2}=.30$. There was a main effect of frame alignment, $F(1,25)=5.80, p=.024, \eta_{p}^{2}=.19$, with better performance in the aligned, than the misaligned, frame 
condition. There was no main effect of face alignment, $F(1,25)$ $=.35, \mathrm{p}=.560, \eta_{\mathrm{p}}^{2}=.01$. However, there was a marginally significant interaction between congruency and face alignment, $F(1,25)=4.11, p=.053, \eta_{p}^{2}=.14$ (see Fig. 3B).

Planned paired t-tests (2-tailed) performed on response time (RT) indices of holistic processing $\left(\mathrm{RT}_{\text {congruent }}-\right.$ $\mathrm{RT}_{\text {incongruent }}$ ) failed to reveal any significant difference in holistic processing when both the face parts and the frames were misaligned compared to when they were both aligned, $\mathrm{t}(25)=$ $0.58, \mathrm{p}=0.566$, Hedges's $\mathrm{g}_{\mathrm{av}}=.15$. There was also no significant difference in holistic processing when just the frame was misaligned (i.e., aligned face/misaligned frame condition), $\mathrm{t}(25)=1.16, \mathrm{p}=0.257$, Hedges's $\mathrm{g}_{\mathrm{av}}=.33$ ), or just the face was misaligned, $\mathrm{t}(25)=1.40, \mathrm{p}=0.175$, Hedges's $\mathrm{gav}_{\mathrm{av}}=$ .46 , relative to when both the face parts and frames are aligned, although there was a trend towards a cost in the later case. Notably, indices of holistic perception in both of these conditions, that is, where only the frame, $\mathrm{t}(25)=1.37, \mathrm{p}=$ 0.184 , Hedges's $g_{a v}=.40$, or only the faces parts where misaligned, $\mathrm{t}(25)=0.87, \mathrm{p}=.392$, Hedges's $\mathrm{g}_{\mathrm{av}}=.25$, also did not differ from that when both the face and frame were misaligned, as in the standard composite face misaligned condition. Thus, there is no statistical evidence of a speed accuracy trade-off occurring in the data from Experiment 2.

As in Experiment 1, performance in the isolated part condition generally fell numerically between performance in the conditions where a congruent or an incongruent taskirrelevant part appeared for both sensitivity (d') and RT measures (see Fig. 3).

\section{Discussion}

Experiment 2 provided a replication of the findings from Experiment 1: misaligning the framing contour of the face disrupted holistic processing, even when the configuration of the internal features remained intact. However, presenting a face with a disrupted configuration in an intact face frame did not preserve holistic face processing. The disruption to holistic processing when just the internal features were misaligned was equivalent to that when both the internal features and external face frame were misaligned. Thus, while a disrupted face frame can disrupt holistic perception of faces, an intact frame cannot support holistic perception in the context of a face with a disrupted configuration.

The slightly smaller size of the effect of misaligning the framing contour of the face on holistic processing in Experiment 2 appears to be a result of increased variability in the data as numerically the size of the cost is similar across the experiments. It is possible that the introduction of the condition where the face parts were misaligned, but presented in aligned frames, created a disruptive task context for the other conditions as these faces have a strange and almost grotesque appearance.
The inability of the intact frame in Experiment 2 to support holistic perception of a face with a disrupted configuration suggests that the disruption to holistic perception in the misaligned condition in the composite face task is not entirely driven by the disrupted external contour that results from this manipulation. Also noteworthy is that, in both Experiment 1 and Experiment 2, the level of holistic perception of faces with an intact internal configuration but distracted external contour, sits numerically at an intermediate level between the condition with both configuration and external contour intact and that with both disrupted. Thus, both intact perceptual grouping and intact facial configuration appear necessary for normal holistic perception to occur.

\section{General discussion}

The studies reported here provide converging evidence of the importance of general object processing mechanisms for holistic perception, as indexed via the composite face task. As predicted, holistic perception was impaired when the Gestalt grouping cue of good continuation was disrupted by placing the face in a misaligned frame. This finding suggests that the disrupted contour that results from misaligning the top and bottom parts of composite faces can serve as an important feature contributing to the reduced holistic processing of misaligned faces. This finding is consistent with our previous demonstration that disrupting the perceptual grouping of the top and bottom face parts, in that case by presenting the top and bottom face parts on misaligned and differently colored backgrounds, impairs holistic perception (Curby et al., 2016; Curby et al., 2013). Notably, these data extend our previous findings by adopting a more direct manipulation to disrupt the perceptual grouping of the face parts, while again leaving the configuration of facial features intact. Specifically, while our previous study manipulated contextual (background) cues that supported or impaired perceptual grouping, our current study manipulated cues to disrupt the objecthood of the face that could be perceived as part of the stimulus itself: that is, its external contour.

In addition, by including the standard misaligned condition, these data extend our previous findings by providing a direct comparison of the degree to which disrupting perceptual grouping, compared to disrupting the configuration of the face, drives the robust and widely demonstrated cost to holistic perception with part misalignment. Further, the current data provides important new insights into this classic misalignment effect that is the cornerstone of our understanding of holistic face perception. Our findings suggest that the disruption to the face template may only partially account for the drop in holistic perception with part misalignment. Instead, our findings suggest that the disruption to the objecthood of the face, which occurs as a consequence of misalignment, plays a central role. 
The likely importance of the disruption to the external contour in driving the attenuation of holistic processing observed with misalignment has also been noted previously in the literature (Rossion, 2013). Laguesse and Rossion (2013) examined whether it was likely the introduction of the discontinuity in the external contour, which causes a "breaking" of the whole, or the increase in metric distances between important facial features (e.g., the eyes and mouth) that underlies the drop in holistic processing with misalignment. They parametrically manipulated the degree of misalignment (and thus the change in distances between the features) and found similar effects regardless of degree of misalignment. Consistent with the findings reported here, Rossion (2013) suggested that it is the ability to perceptually integrate the face parts that is critical in driving the misalignment effect, rather than the changes to distances between features. Further, we suggest that this perceptual integration of the facial features may oblige observers to select the face as a perceptual unit, contributing to the holistic effects observed in the composite face task.

Our findings also appear to provide support for the importance of (intact) facial configuration in supporting holistic face perception: holistic perception was similarly attenuated for faces with a misaligned configuration, regardless of whether they were presented with an intact or disrupted external contour. However, the potential mechanistic basis of the impact of disrupting the familiar facial configuration on holistic perception is not clear. Some possibilities are discussed below.

The impact of experience on perceptual grouping and object-based attention renders it difficult to fully tease apart the relative contribution of the familiar face configuration and perceptual grouping in supporting holistic face perception. Experience can establish and/or strengthen groupings between parts, resulting in stronger object-based attention for familiar than unfamiliar shapes and orientations (Vecera \& Farah, 1997; Zemel, Mozer, Behrmann, \& Bavelier, 2002). Specifically, highly familiar stimuli, such as letters, have been shown to exhibit markers of strong objecthood. For example, image segmentation, a critical precursor for objecthood, is superior for upright, compared to inverted, letters (Vecera \& Farah, 1997). Our extensive experience with faces may render them into highly coherent units obliging object-based selection. These qualities - strong grouping between features and the resulting cohesive object-based representations - may facilitate interactive encoding of features and their object context (i.e., "holistic" effects) and underlie demonstrated failures of selective attention to features within faces. Thus, given evidence that familiarity and experience can facilitate grouping, our extreme familiarity with the classic (upright) face configuration may be integral in supporting the proposed strong objecthood of faces and thus the coherence of the face as a perceptual unit. Disrupting the configuration of facial features may impair this benefit afforded to highly familiar objects, thereby weakening the grouping and coherence of the face as stimulus.

On a related note, disrupting the configuration of the internal features likely also disrupted the objecthood of the face at a stimulus level, even when the external contour remained intact. An unfortunate consequence of misaligning the internal facial features is a disruption to the internal contours within the face. These contours also likely support the grouping of the facial features. That is, despite the external framing contour being intact, a number of other, internal contours are disrupted when the face parts are misaligned. For example, the contour of the bridge of the nose and the convex contour of the cheeks is disrupted when the face parts are misaligned. It is possible, even likely, that the disruption to the good continuation of the internal face contours served to impede the automatic spreading of attention to, or prioritization of, the other, task-irrelevant, part of the face. Thus, it is unclear to what degree the reduced holistic perception of the misaligned face parts presented with an intact external contour was driven by the disruption to the configuration or to the perceptual grouping of the face parts.

Recent work by Zhao and colleagues is consistent with a disrupted perceptual grouping account of the impaired holistic perception of misaligned faces, even when they are presented in an aligned frame. This work highlighted the potential role of the strong Gestalt grouping within face stimuli as a factor driving holistic face perception by revealing face-like holistic perception for non-face stimuli strong in Gestalt grouping cues (Zhao, Bulthoff, \& Bulthoff, 2016). Given that countless stimuli possess an external contour with good continuation, it is unlikely that this perceptual grouping cue alone supports the strong grouping present in face stimuli. Thus, the internal shape information (e.g., contours) within faces is also likely to contribute to the strong perceptual grouping within faces. It may not be surprising that holistic perception is impaired when these internal grouping cues are disrupted, despite being presented in an intact external frame (contour).

It is possible that holistic perception involves face-specific mechanisms that are tied to facial configuration and that such mechanisms require perceptual grouping to be intact to operate. Specifically, while disrupting perceptual grouping would impair holistic perception, intact perceptual grouping in the presence of a disrupted facial configuration might be insufficient to support holistic face perception. Thus, perceptual grouping might operate independently of holistic perception, but it must be intact for holistic perception to occur. Further studies are required to explore this possibility.

In sum, the findings reported here raise a number of questions and suggest many avenues for future research. While they provide converging evidence of the importance of intact perceptual grouping for holistic face perception, they also reveal how disrupting the internal face configuration impairs holistic perception. However, the mechanistic basis by which 
disruptions of internal face configuration impact holistic processing remains an open question. Future studies should examine, for example, whether it is also linked with perceptual grouping via the discontinuities to the internal contours of the face introduced when the facial configuration was changed, or alternatively whether facial configuration more directly supports holistic perception via providing access to an upright face template necessary for holistic perception.

In conclusion, our findings support the suggestion that the disruption to "objecthood" with face part-misalignment contributes to the classic misalignment effect on holistic processing of faces. Disrupting the perception of the face as a perceptual unit, whether it be via introducing a discontinuity in the external frame or contextual cues that break the perceptual grouping of the face parts (as we did previously), disrupts holistic face perception. Future work should aim to understand if and how perceptual grouping and configuration work together to produce holistic processing effects.

Acknowledgments This research was supported by a grant from the Australian Research Council (ARC) (DE130100969) to KMC. We would also like to thank Denise Moerel for help with data collection for Experiment 2.

\section{References}

Curby, K. M., Entenman, R. J., \& Fleming, J. (2016). Holistic face perception is modulated by experience-dependent perceptual grouping. Attention, Perception, \& Psychophysics, March 30th. [Epub ahead of print]

Curby, K. M., Goldstein, R. R., \& Blacker, K. (2013). Disrupting perceptual grouping of face parts impairs holistic face processing. Attention, Perception, \& Psychophysics., 75(1), 83-91.

Curby, K. M., Johnson, K. J., \& Tyson, A. (2012). Face to face with emotion: Holistic face processing is modulated by emotional state. Cognition and Emotion, 26(1), 93-102.

Diamond, R., \& Carey, S. (1986). Why faces are and are not special: An effect of expertise. Journal of Experimental Psychology: General, 115(2), 107-117.

Egly, R., Driver, J., \& Rafal, R. D. (1994). Shifting visual attention between objects and locations: Evidence from normal and parietal lesion subjects. Journal of Experimental Psychology: General, 123(2), 161-177.

Farah, M. J., Wilson, K. D., Drain, M., \& Tanaka, J. W. (1998). What is "special" about face perception? Psychological Review, 105(3), $482-498$.

Fredrickson, B. L., \& Branigan, C. (2005). Positive emotions broaden the scope of attention and thought-action repertoires. Cognition \& Emotion, 19(3), 313-332.

Gauthier, I., Curran, T., Curby, K. M., \& Collins, D. (2003). Perceptual interference supports a non-modular account of face processing. Nature Neuroscience, 6(4), 428-432.

Hollingworth, A., Maxcey-Richard, A. M., \& Vecera, S. P. (2012). The spatial distribution of attention within and across objects. Journal of Experimental Psychology: Human Perception and Performamce, $38(1), 135-151$.

Kramer, A. F., \& Jacobson, A. (1991). Perceptual organization and focused attention: The role of objects and proximity in visual processing. Perception \& Psychophysics, 50(3), 267-284.
Laguesse, R., \& Rossion, B. (2013). Face perception is whole or none: Disentangling the role of spatial contiguity and interfeature distances in the composite face illusion. Perception, 42(10), 1013-1026.

Lakens, D. (2013). Calculating and reporting effect sizes to facilitate cumulative science: A practical primer for t-tests and ANOVAs. Frontiers in Psychology, 4, 863.

Lundqvist, D., Flykt, A., \& Ohman, A. (1998). The karolinska directed emotional faces-KDEF.: CD-ROM from department of clinical neuroscience, psychology section, Karolinska institutet. Sweden: Stockholm.

Matsukura, M., \& Vecera, S. P. (2006). The return of object-based attention: Selection of multiple-region objects. Perception \& Psychophysics, 68(7), 1163-1175.

McKone, E., Kanwisher, N., \& Duchaine, B. C. (2007). Can generic expertise explain special processing for faces? Trends in Cognitive Science, 11(1), 8-15.

Nakayama, K., Shimojo, S., \& Silverman, G. H. (1989). Stereoscopic depth - its relation to image segmentation, grouping, and the recognition of occluded objects. Perception, 18(1), 55-68.

Palmer, S. E. (1977). Hierarchical structure in perceptual representation. Cognitive Science, 9, 441-474.

Rhodes, G., \& Jeffery, L. (2006). Adaptive norm-based coding of facial identity. Vision Research, 46(18), 2977-2987.

Richard, A. M., Lee, H., \& Vecera, S. P. (2008). Attentional spreading in object-based attention. Journal of Experimental Psychology: Human Perception \& Performance, 34(4), 842853.

Richler, J. J., Bukach, C. M., \& Gauthier, I. (2009). Context influences holistic processing of nonface objects in the composite task. Attention, Perception, \& Psychophysics, 71(3), 530-540.

Richler, J. J., Tanaka, J. W., Brown, D. D., \& Gauthier, I. (2008). Why does selective attention to parts fail in face processing? Journal of Experimental Psychology: Learning, Memory, \& Cognition, 34(6), $1356-1368$.

Rossion, B. (2013). The composite face illusion: A whole window into our understanding of holistic face perception. Visual Cognition, 21(2), 139-253.

Shomstein, S., \& Yantis, S. (2002). Object-based attention: Sensory modulation or priority setting? Perception \& Psychophysics, 64(1), 4151.

Tanaka, J. W., \& Farah, M. J. (1993). Parts and wholes in face recognition. Quarterly Journal of Experimental Psychology A, 46(2), 225245.

Tanaka, J. W., \& Sengco, J. A. (1997). Features and their configuration in face recognition. Memory \& Cognition, 25(5), 583-592.

Taubert, J., \& Alais, D. (2009). The composite illusion requires composite face stimuli to be biologically plausible. Vision Research, 49(14), $1877-1885$.

Tsao, D. Y., \& Livingstone, M. S. (2008). Mechanisms of face perception. Annual Review of Neuroscience, 31, 411-437.

Vecera, S. P., \& Farah, M. J. (1997). Is visual image segmentation a bottom-up or an interactive process? Perception \& Psychophysics, 59(8), 1280-1296.

Yin, R. K. (1969). Looking at upside-down faces. Journal of Experimental Psychology, 81(1), 141-145.

Young, A. W., Hellawell, D., \& Hay, D. (1987). Configural information in face perception. Perception, 10, 747-759.

Zemel, R. S., Mozer, M. C., Behrmann, M., \& Bavelier, D. (2002). Experience-dependent perceptual grouping and object-based attention. Journal of Experimental Psychology: Human Perception and Performance, 28(1), 202-217.

Zhao, M., Bulthoff, H. H., \& Bulthoff, I. (2016). Beyond faces and expertise: Facelike holistic processing of nonface objects in the absence of expertise. Psychological Science, 27(2), 213-222. 